Article

\title{
Feasibility of a multicomponent training for people with mod- erate to severe dementia living in a long-term care home - so- cial ethical approach
}

\author{
Adele Kruse 1, Thomas Cordes ${ }^{1}$, Steffen Schulz 1, PD Dr. Bettina Wollesen 1,* \\ Department of Human Movement Science, University of Hamburg, Germany \\ * Correspondence: bettina.wollesen@uni-hamburg.de; Tel.: +49 (0)30 314-79 477
}

\begin{abstract}
Multicomponent training is recommended for people with dementia living in long-term care homes. Nevertheless, evidence is limited and people with severe dementia are often excluded from trials. Hence, the aim of this study was to investigate (1) the feasibility and (2) the requirements regarding a multicomponent training for people with moderate to severe dementia. The study was conducted as an uncontrolled single arm pilot study with a mixed methods approach. 15 nursing home residents with a mean age of 82 years (range: 75-90 years; female: $64 \%$ ) with moderate to severe dementia received 16 weeks of multicomponent training. Feasibility and requirements of the training were assessed by a standardized observation protocol. Eleven participants regularly attended the intervention. The highest active participation was observed during gait exercises (64\%), the lowest during strength exercises (33\%). It was supportive if exercises were task-specific or related to everyday life. This study confirms that a multicomponent training for the target group is (1) feasible and well accepted. To enhance active participation (2) individual instructions and the implementation of exercises related to everyday life is required. The effectiveness of the adapted training should be tested in future randomized controlled trials.
\end{abstract}

Keywords: Dementia, multicomponent training, long-term care home, social ethical approach

\section{Introduction}

In 201546.8 million people worldwide were living with dementia and this number is estimated to almost double every 20 years [1]. Dementia, leads to a progressive decline in function of various cognitive domains (e.g. complex attention, executive function, learning and memory, language, perceptual-motor and social cognition) [2,3]. Furthermore, dementia is associated with an increasing need of support in activities of daily living (ADL), loss of mobility and increased risk of falling [2,4,5].

However, regular physical activity (PA), defined as skeletal muscle-initiated body movement that results in energy expenditure [6], can counteract these tendencies. Regular PA has beneficial effects on a person's health and promotes so-called healthy ageing (American College of Sports Medicine, 2009). This includes a reduced loss of function, and improvements in endurance and strength in both healthy people and people with noncommunicable diseases [8]. Besides, PA also improves brain structure and function as well as specific cognitive abilities (Erickson, Hillman, \& Kramer, 2015; Voelcker-Rehage \& Niemann, 2013). PA, however, is significantly reduced in people with dementia when compared to age-sex matched healthy controls [4]. Consequently, it is important to promote PA in older people, regardless of their cognitive abilities. 
Furthermore, training beneficially impacts strength, mobility, and ADL in long-term care home (LTCH) residents with and without dementia [11-15]. Training is defined as structured (frequency, intensity, time, and type - FITT) PA targeting the preservation or enhancement of health-related components (e.g., strength, endurance, mobility, balance) $[6,16]$. Especially, multicomponent training combining strength, endurance, postural and balance exercises is recommended [12,17]. In the context of multicomponent training programs, significant improvements or positive trends in walking ability were identified in LTCH residents [18]. In addition, motor-cognitive exercises are beneficial for improving cognitive and motor performance in older adults $[19,20]$. Since people with dementia show increased walking insecurity, it is particularly important to develop and implement specific exercise programs in this target group [5].

However, evidence for a beneficial impact of (multicomponent) training for people with dementia is limited, as most studies are of moderate or low quality [12,14,21]. Furthermore, precise information on the combination of FITT components are unclear and seldomly reported in exercise programs for LTCH residents [22] and people with dementia $[12,23]$. Additionally, people with advanced cognitive impairment are often excluded from trials as they don't meet the required cognitive status [14]. Since people in the same care unit may differ largely in their cognitive status, this is a highly exclusionary approach in practice. In this context, an inclusive social ethical approach would allow all residents of the same care unit to participate. However, this practical approach needs to be tested for feasibility and acceptance.

To develop an effective exercise program for older adults with dementia living in a LTCH, the residents' requirements and needs as well as the setting should be considered [21,24]. Amongst the presence of a well-known person, the specific communication, one-on-one situations and hands-on instructions are recommended to facilitate active participation [24,25]. Interventions should be individualized to challenge the participants' maximal capacity [15]. Moreover, individualized interventions tend to be more effective in improving the quality of life and other psychosocial components in people with dementia [26].

Although multicomponent training is recommended, the best combination of components is yet to be determined for LTCH residents with moderate to severe dementia. Hence, the critical question is: How should a multicomponent training be structured to be feasible for people with moderate to severe dementia living in a LTCH? Furthermore, which are the study population's requirements for a training intervention? Thus, the aim of the present study was to develop and evaluate the feasibility (1) and requirements regarding instruction methods, exercises and the setting (2) of a multicomponent training for people with moderate to severe dementia living in the same care unit in a LTCH. We assume that a multicomponent training is feasible if the target group's needs are met.

\section{Materials and Methods}

The study was conducted as an uncontrolled single arm pilot study to assess the feasibility of a multicomponent training for LTCH residents with moderate to severe dementia. As a reporting guideline the extended CONSORT statement for feasibility studies [27] was used. The study was approved by the local ethics committee of the faculty of psychology and human movement science of the University of Hamburg (2019_249) and prospectively registered at the German Clinical Trial Register (ID: DRKS00021438).

\subsection{Participants}

Participants were recruited at a LTCH for people with dementia in Northern Germany. This LTCH was part of the Prevention and occupational health in long-term care-project (PROCARE) [28] and a multicomponent training group was already implemented. For this feasibility study we included residents with dementia who, beforehand, were excluded from the pre-existing PROCARE training group because they had difficulties following verbal instructions and/or the inability to concentrate throughout the whole class. The eligibility criteria for the modified training program for people with dementia were voluntary participation, written informed consent of a legal guardian and a diagnosis of moderate to severe dementia given by the respective physician. As this multicomponent training was designed specifically for residents who were not able to participate in the pre-existing PROCARE exercise program, no other inclusion or exclusion criteria was applied. The sample size was set to a maximum of 15 participants which was based on the guidelines for preventive sports by the German National Association of Statutory Health Insurance Funds [29].

The consulting psychologist identified $N=15$ participants who met the inclusion criteria. On average participants were 82 years old (range: 75-90 years; female: 64\%). A participant flowchart is presented in Figure 1.

\subsection{Intervention}

The multicomponent training which was designed for PROCARE (Cordes et al., 2019) was modified to the specific requirements of the target group (cf. Table 1). Each session consisted of five components and was structured as follows:

Table 1. Comparison of the multicomponent training PROCARE and PROCARE dementia 


\begin{tabular}{ccc}
\hline Component & PROCARE original & PROCARE dementia \\
\hline duration & 32 sessions in 16 weeks & 16 sessions in 16 weeks \\
Warm-up & $5-10$ minutes standing & 5 minutes seated \\
balance, coordination and cognition & 10 minutes & $10-15$ minutes \\
Gait exercise & 20 minutes & 5 minutes per person \\
& & If not able to walk: chair-based exercise \\
Strength & 10 minutes & with the nurse \\
Cool Down & $5-10$ minutes & 5 minutes \\
\hline
\end{tabular}

The original exercise program included 32 training sessions. They were conducted over a period of 16 weeks and took place twice a week. For this study training sessions were reduced to a total of 16 sessions because the supervising nurse had many other obligations and could only attend once a week. The sessions were conducted in a period of 16 weeks and took place once a week. Each session lasted for 45 to 60 minutes.

In addition to the original program, specific exercise for people with dementia were added [30]. Most of the exercises were performed while participants were seated to enable all residents to participate. Exercise equipment included gymnastic sticks or soft balls as well as everyday objects like towels (cf. Table 3a). The program was supervised by the researcher (a sports scientist) with the assistance of a nurse known by all participants. The gait exercise was performed individually and accompanied by the sports scientist. Meanwhile, the nurse performed activating exercises with the non-ambulatory participants (like the coordination component). Based on an observation protocol the program was adjusted after each session. This process included optimizing the instruction methods (choice of words, position of the trainer, individual or group approach, haptic/visual/auditive cues) and the adaption of exercises according to the FITT components [31]. Training sessions took place in a recreation room at the care unit.

\subsection{Outcome measures}

The measurements addressed the main aspects of feasibility (adherence, acceptance, exercise quantity, performance quality). Additionally, the participants' age and sex were provided by the facility's psychologist.

\subsubsection{Primary outcome}

Feasibility (1) of the intervention was assessed by means of a standardized observation protocol. For all sessions the retention rate (dropouts, attendance, adverse events) and the number of residents who showed signs of apathy and therefore could not actively participate were documented. In addition, exercise quantity (the number of exercises planned and performed per session) was documented. For eight sessions a research assistant documented the performance quality divided into active ("exercise was performed correctly" or "attempts were made to perform the exercise") and inactive ("no response"). Acceptability was assessed by a four-point Likert scale at the end of each session. Each item contained a statement combined with a smiley face.

\subsubsection{Secondary outcome}

Requirements regarding the instruction methods, the feasibility of exercise and the setting (2) of the intervention were documented in an observation protocol. Half of the sessions and subsequent discussions with the nurse were documented by the sports scientist and the other half by the research assistant. Additionally, the nurse's feedback was obtained through a questionnaire with open-ended questions after the 16-week intervention was completed. 


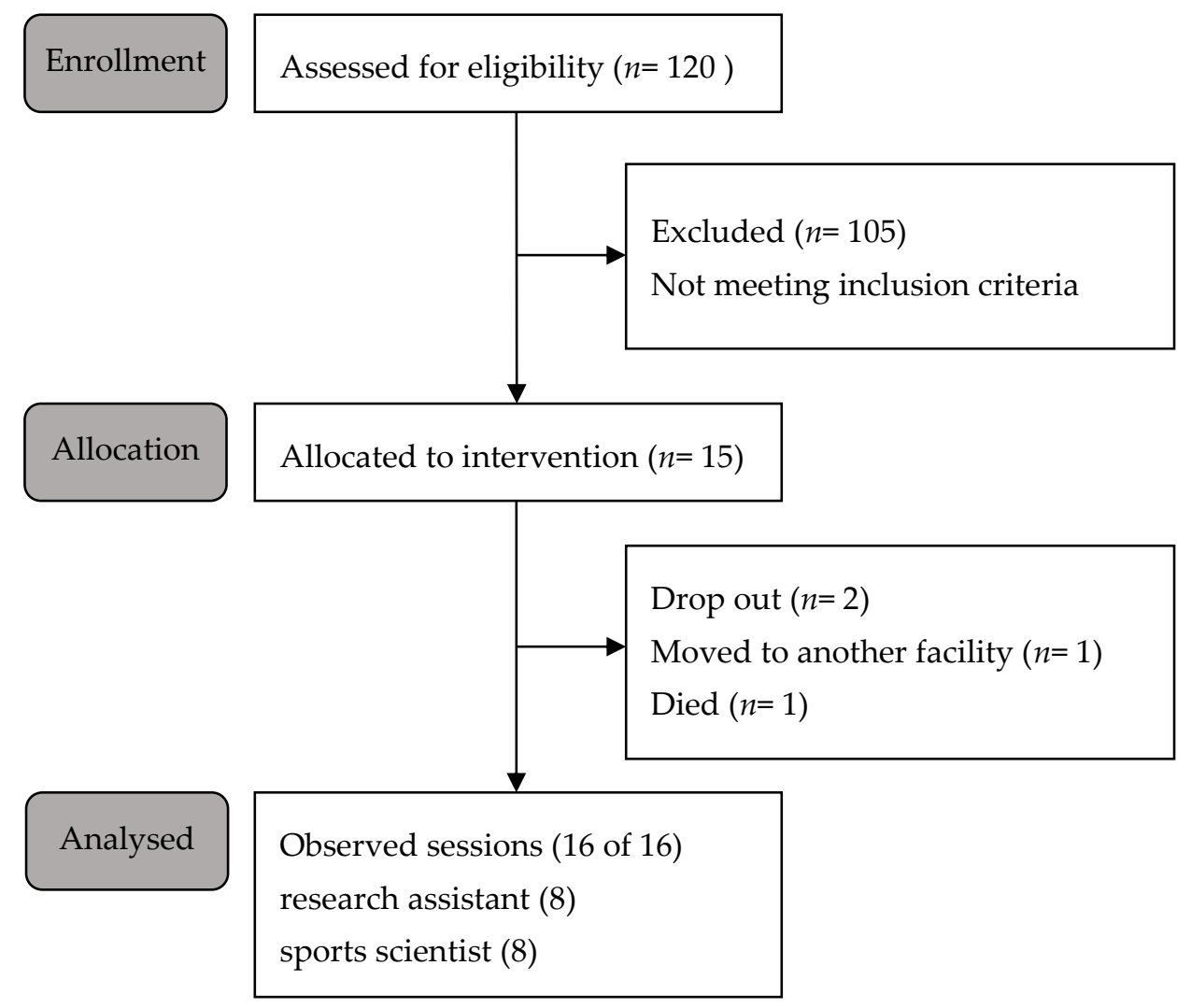

Figure 1. Participant flowchart.

\subsection{Statistics}

Descriptive statistics were used for quantitative measures (attendance and performance quality, exercise quantity). The activity rate (percentage of active participants in relation to all attendees) was calculated for each session and each component. In addition, the proportion of individuals with signs of apathy within the group of inactive participants was calculated. The mean (M) and standard deviation (SD) were calculated for the average number of exercises performed per session (adapted intervention of this study) and planned per session (PROCARE). Qualitative measures (field notes in blank spaces and nurse's questionnaire) were evaluated by content analysis with category formation.

\section{Results}

The mean attendance rate of the 15 participants included in the study was $72 \%$ (attendance ranged between $53 \%$ and $100 \%$ per sessions). Of the eleven regular attendees, six (55\%) were able to actively participate. Three (27\%) regularly attending participants showed signs of severe apathy (cf. Table 2). Two participants observed most of the time instead of taking part. Two participants sometimes left during the sessions and returned later. Reasons for participant absence were visits of relatives/friends, other appointments, or health related. There were no adverse events reported during or relating to the intervention program.

Table 2. Baseline characteristics of participants.

\begin{tabular}{cccc}
\hline & participants & female & male \\
\hline Included & 15 & 10 & 5 \\
Ambulatory & 10 & 5 & 5 \\
Signs of apathy & 3 & 3 & 0 \\
\hline
\end{tabular}




\section{Regularly active}

6

3

3

The participants acceptance could not be assessed as planned. After eight sessions, the survey was stopped because the number of participants able to respond was limited. In addition, participants had difficulties identifying the smiley faces, reading the statements and choosing one.

\subsection{Performance quality and exercise quantity}

The mean activity rate for the eight sessions assessed by the research assistant was $46 \%$ (cf. Table B1). During these sessions an average of $29 \%$ of participants were inactive due to apathy. For the different components the highest activity rate was observed for the gait exercise (63\%). The lowest activity rate was observed during the strength exercises (35\%). (cf. Figure 2)

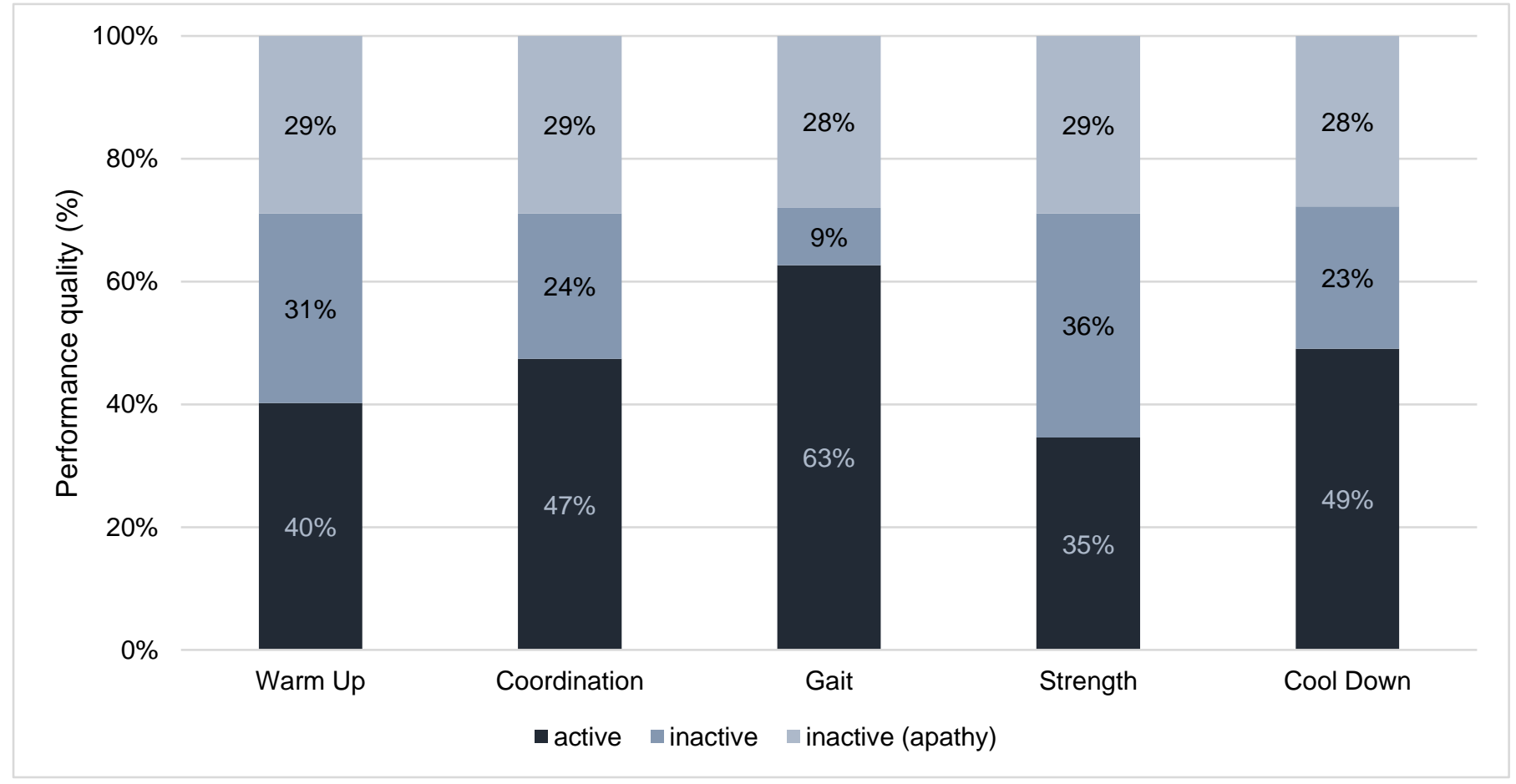

Figure 2. Performance quality (active/inactive/apathy) in percent for each component observed during eight training sessions.

On average, $23(\mathrm{SD} \pm 5)$ exercises were planned for each session, of which only $19.5(\mathrm{SD} \pm 4)$ exercises could be performed as the participants needed time to understand the exercises. The original PROCARE program included a mean of $35(\mathrm{SD} \pm 12)$ exercises per session. In the present study, participants walked an average of $22.5 \mathrm{~m}$ (range $0-30 \mathrm{~m})$ per session, which is $13 \%$ of the distance of the PROCARE intervention $(170.6 \mathrm{~m}$; range $150-240 \mathrm{~m})$.

\subsection{Secondary outcome}

The content analysis of the 16 observation protocols included four main categories ("Instruction Methods", "Exercise Design", "Exercise Modification", "Setting”).

\subsubsection{Instructions}

It was observed that more people actively participated when the general demonstration and pictorial description of the exercise was followed by individual instructions. The attention of individual participants could be increased by addressing them by name with eye contact. When explaining the exercise individually, the sports scientist sat directly in front of the resident and demonstrated the exercise in a mirror image in combination with pictorial description and sometimes tactile cues. The nurse also stated that tactile stimuli are especially important for "persons who may miss the meaning of words" to understand the exercises. The sports scientist used a rolling stool to quickly switch between participants. It was helpful to repeat the instructions several times to keep the participants' attention.

\subsubsection{Exercise design}


In general, the nurse described the program as appealing and well thought out. Additionally, she pointed out positively that an unknown trainer and new activities break up the routine of daily living.

Exercises that related to everyday life (e.g., "use the towel as if you were drying your back") or included a concrete instruction for action (e.g., "look at the neighbor on your right" instead of "turn your head to the right") could be performed by more participants. It was supportive when the movement was performed in the field of vision. Especially throwing and catching activated many people. Performance was particularly high during exercises that were performed in pairs with the sports scientist (e.g., rope pull). The nurse noted that exercises were recognized by some individuals after three to four weeks due to regular repetition. Participants expressed their own ideas for exercises with reference to everyday life, music and equipment. The equipment was generally observed as activating but the size and design of the material also had an influence on the feasibility of exercises. All participants, including those with signs of apathy, held or engaged with cloths and small balls.

\subsubsection{Exercise modification}

Exercises in which body parts were moved alternately, or dual-tasks as well as grabbing and releasing material were difficult for many participants. Particularly difficult were exercises that were not related to everyday life (e.g., "turn your head to the left" or "press your hands against the outside of your thighs and try to open your thighs at the same time"). By providing a concrete action reference (e.g., "look at your left neighbor") or tactile cues (e.g., trainer presses against the outer thigh), the realisation of these exercises were improved. Furthermore, it was difficult for participants to perform exercises without visible movement (keeping a ball squeezed or relaxation exercises like the body scan). It was supportive to repeatedly demonstrate the movement (repeatedly squeezing the ball) and to use active relaxation exercises (tapping massage).

It was observed that fewer repetitions and static exercise were carried out more successfully. When switching between exercises, these had to be explained again each time. The nurse also noted that exercises became feasible through modification and after weekly repetition (three to four weeks). Additionally, she observed that individuals who are harder to reach responded with attention after seven to eight sessions. During the training she observed more "contact looks" between participants. During the course of the training, it was observed that participants needed some time to adjust to the situation at the beginning of the training and after the gait exercise. By integrating motivational exercise equipment, the attention of the participants was refocused.

After the first session, the nurse expressed concerns about the safety of the gait exercises. Possible risk of falling and safety measures to avoid falls were discussed with the nurse, the psychologist, the sports scientist and the research assistant. As a result, it was decided to carry out the gait exercises with one-on-one supervision. It became apparent that it was safer to walk in an arc and not to turn around on the spot at the end of the course.

\subsubsection{Setting}

It was observed that the armrests of the chairs were a hindrance during several exercises. Chairs without armrests would have been helpful for the residents actively participating.

It was observed that the number of active residents affected the activity level of each participant in the group. A greater number of active residents favourably influenced the activity level of habitually less active participants.

It was noted several times that one-on-one supervision would be useful. Additional staff made it possible to activate more participants at the same time. Participants were more active when a relative assisted them throughout the training. The nurse recommended a maximum group size of eight and the presence of a second instructor to enable all participants to be active.

\section{Discussion}

The aim of this study was to investigate (1) the feasibility as well as (2) the requirements regarding instruction methods, exercises design and setting of a multicomponent training for people with moderate to severe dementia. Overall, the multicomponent training program was accepted by most of the participants (mean attendance of $72 \%$ ) which is similar to the adherence rate reported for training programs for people with mild to moderate dementia (mean attendance $70-80 \%$ ) [12]. However, as the participants' opinion could not be assessed this conclusion is limited. Although the use of the faces scale was not feasible in this study, Hendriks and colleagues (2021) successfully assessed the acceptance of an art intervention for people with dementia with the Visual Analog Mood Scale (VAMS, [32]. However, this finding was published subsequent to the completion of the multicomponent training [33]. The scales differ in the design and description of the faces, which is simpler in the VAMS (one explanatory word under each face). Using a similar design and a one-word description instead of complete sentences probably could have increased the feasibility of this selfreport measure. 
In addition to the symptoms of moderate to severe dementia, participants in this study had to cope with several health conditions which is common in this population [34]. Thus, the training had to be adapted to the different levels of performance and attention, including walkability and apathy.

Participant understanding of the exercises was a critical factor throughout the training and across all components. Although this impact was observed multiple times in the present study, the instructional methods are rarely discussed $[12,13,15,21]$. Individualised instruction combining verbal, auditory, visual and tactile cues were most effective which is in line with recommended communication methods in dementia care (Eggenberger et al., 2013). This was further facilitated in this study by the trainer sitting on a rolling stool and changing position frequently. In order to ensure an individual approach right from the start it would have been advisable to consult the nurse in advance. However, in this study interdisciplinary teamwork began with a discussion following the third training session. Consequently, the first three training sessions focused on familiarization. The individual instruction as well as one-on-one support facilitated the engagement of more residents. This is also reflected in the training components, where the highest activity was observed during gait exercises, which were performed with one-on-one support. However, these methods are very time-consuming, which explains the low exercise quantity. In contrast to PROCARE ( $m=35 ; \mathrm{SD} \pm 12)$, considerably fewer exercises could be performed $(\mathrm{m}=20 ; \mathrm{SD} \pm 4)$. This difference is highlighted through the gait exercise, resulting in a considerably lower intensity (0-30 m per session) than in the PROCARE program (150-240m per session; Cordes et al., 2019). Due to fewer time, the intensity decreased as the proportion of ambulatory residents in the group increased. In order to integrate gait exercises and still provide an adequate intensity for both ambulatory and non-ambulatory participants, tailored chair-based exercises should be integrated for those who are unable to walk [22]. To implement those simultaneous exercises, at least one trainer and one caregiver (e.g., nurse) are required. In addition, it was observed that the support of relatives during the exercises was activating. The increased activity of residents supported by their relatives during the exercises was also described by van Alphen and colleagues (2016). For adequate instruction according to the previously described instruction methods, the group size should be limited. With an average of eleven residents, the group was larger than in comparable studies (two to seven participants) [12]. A smaller group size of eight participants was also suggested by the nurse.

Additionally, there is repeated emphasis in dementia research on the need to identify an appropriate configuration of the FITT components with respect to the stage of the disease $[12,14]$. In this study it was found that adapting these components to the needs of people with severe dementia improved the performance quality. For example, multi-set training was found to be difficult to implement because the exercises had to be explained again in each set. In contrast to multi-set training with short time per exercise, single-set training provides more continuous time per exercise. With more continuous time spent on each exercise, it was possible to instruct more participants individually. Hence, the level of active participation increased and individually more exercises were performed.

In addition to identifying the best combination of FITT components [12], this study examined the exercise design. Exercises that could be well implemented related to everyday life or were task-oriented. Furthermore, exercises that were initially not feasible could be modified with regard to these components to increase feasibility. In this context the high activity level during gait exercise - even though there is increasing gait insecurity (Taylor et al., 2019) - could be attributed to its closeness to the daily life of ambulatory residents. In contrast, the low activity observed during strength training may reflect that these exercises were poorly related to ADL. Therefore, strength exercises in future exercise programs should be more task-oriented and related to residents' everyday life activities, even when performed in a sitting position.

Due to the socio-ethical approach of this study, resulting in a very heterogenic group, it is essential to classify the participation rate in a differentiated way. Considering the mean active participation of $46 \%$ relative to $29 \%$ inactivity and $25 \%$ inactivity due to apathy indicates that the majority of residents without apathy participated actively. Nevertheless, a more precise scale would have been useful to quantify the heterogeneous activity levels and thereby take into account the individual capacity of the residents. As a consequence, engagement with equipment (holding, rope pulling with the trainer) and social interaction ("contact looks") could have been included as a level of active participation as this can be considered a success for residents with signs of apathy.

Finally, from a scientific perspective, training can be adapted and targeted more specifically to a homogeneous group. In practice, however, such an approach is highly exclusionary to many residents. Thus, the social ethical approach of this study is relevant to practice. In fact, it was observed that the activity level of the less active individuals was increased by the participation of the more active individuals in the heterogeneous group of this study.

\section{Conclusion}


This study confirms that a multicomponent training for people with moderate to severe dementia is feasible. Since a highly individualized approach seems to increase the level of activity, the group size should be kept to a minimum of eight participants. For adequate supervision of the training an appropriately qualified instructor (e.g., sports scientist) assisted by a caretaker known to the participants are needed. To enable as many LTCH residents as possible to actively participate, exercises should be task-oriented, related to everyday activities and tailored to the individual residents' capacities. Further research should implement the identified requirements of this study to test the effectiveness of this optimized multicomponent training in future randomized controlled trials.

\section{Patents}

Author Contributions: Conceptualization, Adele Kruse, Steffen Schulz and Bettina Wollesen; methodology, Adele Kruse, Steffen Schulz and Bettina Wollesen; investigation, Adele Kruse and Steffen Schulz; data curation, Adele Kruse.; writing-original draft preparation, Adele Kruse; writing - review and editing, Thomas Cordes and Bettina Wollesen; visualization, Adele Kruse and Thomas Cordes.; supervision, Bettina Wollesen; project administration, Bettina Wollesen.; funding acquisition, Bettina Wollesen. All authors have read and agreed to the published version of the manuscript.

Funding: This study was funded by the health insurance Techniker Krankenkasse. The views expressed in this paper are those of the authors and may not be shared by the funding bodies. The study is part of the project "Prevention and occupational health in long-term care" (PROCARE). Data was analyzed independently of the trial sponsors. This funder did not play any role in the design of the study, data analysis, reporting of results, or the decision to present the manuscript for publication.

Institutional Review Board Statement: The study was conducted according to the guidelines of the Declaration of Helsinki, and approved by the Ethics Committee of the Faculty of Psychology and Human Movement, University of Hamburg, Germany (2019_249).

Informed Consent Statement: All participants and their legal guardians were informed about the study goals. Written informed consent was obtained from all participants or their legal guardians prior to the study enrollment according to the Declaration of Helsinki. Participants as well as their relatives or legal guardians were able to withdraw consent at any time.

Data Availability Statement: The data presented in this study are available on request from the corresponding author (BW).

Conflicts of Interest: The authors declare no conflict of interest. 


\section{Appendix A}

Table A1. Exercise examples of a training session with progression of exercises.

\begin{tabular}{|c|c|c|c|}
\hline Training components & Level 1 & Level 2 & Level 3 \\
\hline $\begin{array}{l}\text { Mobilisation and } \\
\text { warm-up }\end{array}$ & $\begin{array}{l}\text { Moderate mobility and } \\
\text { range of motion exercises } \\
\text { for the wrists, hip, } \\
\text { shoulders, knees, and ankles }\end{array}$ & $\begin{array}{l}\text { Moderate mobility and } \\
\text { range of motion exercises } \\
\text { for the wrists, hip, } \\
\text { shoulders, knees, and ankles }\end{array}$ & $\begin{array}{l}\text { Moderate mobility and } \\
\text { range of motion exercises } \\
\text { for the wrists, hip, } \\
\text { shoulders, knees, and ankles }\end{array}$ \\
\hline \multirow{2}{*}{$\begin{array}{l}\text { Coordination and } \\
\text { motor-cognitive } \\
\text { exercises }\end{array}$} & $\begin{array}{l}\text { waving, swinging back and } \\
\text { forth, circling in the air with } \\
\text { a cloth }\end{array}$ & $\begin{array}{c}\text { throw up a cloth and catch } \\
\text { it again }\end{array}$ & $\begin{array}{l}\text { pass cloths to the trainer } \\
\text { and catch them again }\end{array}$ \\
\hline & $\begin{array}{c}\text { cognitive-motor tales } \\
\text { (incorporated movement } \\
\text { into stories e.g., a day at the } \\
\text { long-term care home) }\end{array}$ & $\begin{array}{c}\text { cognitive-motor tales } \\
\text { (incorporated movement } \\
\text { into stories e.g., a visit to the } \\
\text { garden) }\end{array}$ & $\begin{array}{c}\text { cognitive-motor tales } \\
\text { (incorporated movement } \\
\text { into stories e.g., a trip to the } \\
\text { beach) }\end{array}$ \\
\hline \multirow{2}{*}{ Gait exercise } & $\begin{array}{l}15 \mathrm{~m} \text { walk, pleasant pace, } \\
\text { there and back }\end{array}$ & $\begin{array}{l}\text { 15m walk, pleasant pace, } \\
\text { there and back }\end{array}$ & $\begin{array}{l}\text { 15m walk, pleasant pace, } \\
\text { there and back with } \\
\text { instruction "stop and go" }\end{array}$ \\
\hline & & $15 \mathrm{~m}$ walk at a brisk pace & $\begin{array}{c}15 \mathrm{~m} \text { walk at a brisk pace } \\
\text { with instruction "stop and } \\
\text { go" }^{\prime \prime}\end{array}$ \\
\hline \multirow{5}{*}{$\begin{array}{l}\text { Strength and } \\
\text { aerobic exercises }\end{array}$} & bending and stretching & bending and stretching & bending and stretching \\
\hline & $\begin{array}{l}\text { knees, } 20 \text { repetitions } \\
\text { rowing with stick, } \\
15 \text { repetitions }\end{array}$ & $\begin{array}{l}\text { knees, } 2 \times 20 \text { repetitions } \\
\text { rowing with stick, } \\
2 \times 15 \text { repetitions }\end{array}$ & $\begin{array}{l}\text { knees, } 3 \times 15 \text { repetitions } \\
\text { rowing with stick, } \\
3 \times 15 \text { repetitions }\end{array}$ \\
\hline & $\begin{array}{c}\text { rotation of upper body, } \\
10 \text { repetitions }\end{array}$ & $\begin{array}{l}\text { rotation of upper body, } \\
2 \times 15 \text { repetitions }\end{array}$ & $\begin{array}{l}\text { rotation of upper body, } \\
3 \times 15 \text { repetitions }\end{array}$ \\
\hline & $\begin{array}{l}\text { strength exercises with the } \\
\text { towel (e.g., compress } \\
\text { between knees) } 10 \times 3 \mathrm{sec} \text {. }\end{array}$ & $\begin{array}{l}\text { strength exercises with the } \\
\text { towel, } 10 \times 5 \mathrm{sec} \text {. }\end{array}$ & $\begin{array}{l}\text { strength exercises with the } \\
\text { towel, } 15 \times 5 \mathrm{sec} \text {. }\end{array}$ \\
\hline & $\begin{array}{l}\text { Biceps Curls and making a } \\
\text { fist, } 1 \times 15 \text { repetitions }\end{array}$ & $\begin{array}{l}\text { Biceps Curls and making a } \\
\text { fist, } 2 \times 15 \text { repetitions }\end{array}$ & $\begin{array}{l}\text { Biceps Curls } 1-2 \mathrm{~kg} \text { weights, } \\
2 \times 15 \text { repetitions }\end{array}$ \\
\hline Cool down & $\begin{array}{l}\text { stretching exercises and } \\
\text { progressive muscle relaxa- } \\
\text { tion } \\
\end{array}$ & $\begin{array}{c}\text { stretching exercises and } \\
\text { progressive muscle relaxa- } \\
\text { tion } \\
\end{array}$ & $\begin{array}{c}\text { stretching exercises and } \\
\text { progressive muscle relaxa- } \\
\text { tion }\end{array}$ \\
\hline
\end{tabular}

\section{Appendix B}

Table B1. Mean activity rate (\%) for observed sessions divided into four categories.

\begin{tabular}{ccccc}
\hline & active & partially active & inactive & inactive (apathy) \\
\hline Activity (total) & $31 \%$ & $15 \%$ & $25 \%$ & $29 \%$ \\
Warm up & $24 \%$ & $17 \%$ & $31 \%$ & $29 \%$ \\
Coordination & $28 \%$ & $20 \%$ & $24 \%$ & $29 \%$ \\
Gait Exercise & $54 \%$ & $9 \%$ & $9 \%$ & $28 \%$ \\
Strength Exercise & $27 \%$ & $8 \%$ & $36 \%$ & $29 \%$ \\
Cool Down & $23 \%$ & $26 \%$ & $23 \%$ & $28 \%$
\end{tabular}




\section{References}

1. Alzheimer's Disease International World Alzheimer Report 2015, The Global Impact of Dementia: An Analysis of Prevalence, Incidence, Cost and Trends; Alzheimer's Disease International: London, 2015;

2. Sachdev, P.S.; Mohan, A.; Taylor, L.; Jeste, D.V. DSM-5 and Mental Disorders in Older Individuals: An Overview. Harv Rev Psychiatry 2015, 23, 320-328, doi:10.1097/HRP.0000000000000090.

3. World Health Organization Global Action Plan on the Public Health Response to Dementia: 2017-2025; 2017;

4. Hartman, Y.A.W.; Karssemeijer, E.G.A.; van Diepen, L.A.M.; Olde Rikkert, M.G.M.; Thijssen, D.H.J. Dementia Patients Are More Sedentary and Less Physically Active than Age- and Sex-Matched Cognitively Healthy Older Adults. Dement Geriatr Cogn Disord 2018, 46, 81-89, doi:10.1159/000491995.

5. Taylor, M.E.; Brodie, M.A.; van Schooten, K.S.; Delbaere, K.; Close, J.C.T.; Payne, N.; Webster, L.; Chow, J.; McInerney, G.; Kurrle, S.E.; et al. Older People with Dementia Have Reduced Daily-Life Activity and Impaired Daily-Life Gait When Compared to Age-Sex Matched Controls. JAD 2019, 71, S125-S135, doi:10.3233/JAD-181174.

6. Caspersen, C.J.; Powell, K.E.; Christenson, G.M. Physical Activity, Exercise, and Physical Fitness: Definitions and Distinctions for Health-Related Research. Public Health Rep 1985, 100, 126-131.

7. American College of Sports Medicine; Chodzko-Zajko, W.J.; Proctor, D.N.; Fiatarone Singh, M.A.; Minson, C.T.; Nigg, C.R.; Salem, G.J.; Skinner, J.S. American College of Sports Medicine Position Stand. Exercise and Physical Activity for Older Adults. Med Sci Sports Exerc 2009, 41, 1510-1530, doi:10.1249/MSS.0b013e3181a0c95c.

8. Pedersen, B.K.; Saltin, B. Exercise as Medicine - Evidence for Prescribing Exercise as Therapy in 26 Different Chronic Diseases. Scand J Med Sci Sports 2015, 25, 1-72, doi:10.1111/sms.12581.

9. Erickson, K.I.; Hillman, C.H.; Kramer, A.F. Physical Activity, Brain, and Cognition. Curr. Opin. Behav. Sci. 2015, 4, 27-32, doi:10.1016/j.cobeha.2015.01.005.

10. Voelcker-Rehage, C.; Niemann, C. Structural and Functional Brain Changes Related to Different Types of Physical Activity across the Life Span. Neurosci. Biobehav. Rev. 2013, 37, 2268-2295, doi:10.1016/j.neubiorev.2013.01.028.

11. Bischoff, L.L.; Cordes, T.; Meixner, C.; Schoene, D.; Voelcker-Rehage, C.; Wollesen, B. Can Cognitive-Motor Training Improve Physical Functioning and Psychosocial Wellbeing in Nursing Home Residents? A Randomized Controlled Feasibility Study as Part of the PROCARE Project. Aging Clin Exp Res 2020, doi:10.1007/s40520-020-01615-y.

12. Borges-Machado, F.; Silva, N.; Farinatti, P.; Poton, R.; Ribeiro, Ó.; Carvalho, J. Effectiveness of Multicomponent Exercise Interventions in Older Adults With Dementia: A Meta-Analysis. The Gerontologist 2020, gnaa091, doi:10.1093/geront/gnaa091.

13. Demurtas, J.; Schoene, D.; Torbahn, G.; Marengoni, A.; Grande, G.; Zou, L.; Petrovic, M.; Maggi, S.; Cesari, M.; Lamb, S.; et al. Physical Activity and Exercise in Mild Cognitive Impairment and Dementia: An Umbrella Review of Intervention and Observational Studies. J. Am. Med. Dir. Assoc 2020, 21, 1415-1422.e6, doi:10.1016/j.jamda.2020.08.031.

14. Forbes, D.; Forbes, S.C.; Blake, C.M.; Thiessen, E.J.; Forbes, S. Exercise Programs for People with Dementia. Cochrane Database Syst. Rev. 2015, doi:10.1002/14651858.CD006489.pub4.

15. Yeh, S.-W.; Lin, L.-F.; Chen, H.-C.; Huang, L.-K.; Hu, C.-J.; Tam, K.-W.; Kuan, Y.-C.; Hong, C.-H. High-Intensity Functional Exercise in Older Adults with Dementia: A Systematic Review and Meta-Analysis. Clin Rehabil 2020, 026921552096163, doi:10.1177/0269215520961637.

16. ACSM's Guidelines for Exercise Testing and Prescription; American College of Sports Medicine, Riebe, D., Ehrman, J.K., Liguori, G., Magal, M., Eds.; Tenth edition.; Wolters Kluwer: Philadelphia, 2018; ISBN 978-1-4963-3906-5.

17. de Souto Barreto, P.; Morley, J.E.; Chodzko-Zajko, W.; H. Pitkala, K.; Weening-Djiksterhuis, E.; Rodriguez-Mañas, L.; Barbagallo, M.; Rosendahl, E.; Sinclair, A.; Landi, F.; et al. Recommendations on Physical Activity and Exercise for Older Adults Living in Long-Term Care Facilities: A Taskforce Report. J. Am. Med. Dir. Assoc 2016, 17, 381-392, doi:10.1016/j.jamda.2016.01.021. 
18. Arrieta, H.; Rezola-Pardo, C.; Gil, S.M.; Irazusta, J.; Rodriguez-Larrad, A. Physical Training Maintains or Improves Gait Ability in Long-Term Nursing Home Residents: A Systematic Review of Randomized Controlled Trials. Maturitas 2018, 109, 45-52, doi:10.1016/j.maturitas.2017.12.003.

19. Wollesen, B.; Wildbredt, A.; van Schooten, K.S.; Lim, M.L.; Delbaere, K. The Effects of Cognitive-Motor Training Interventions on Executive Functions in Older People: A Systematic Review and Meta-Analysis. Eur. Rev. Aging Phys. Act 2020, 17, 9 , doi:10.1186/s11556-020-00240-y.

20. Wollesen, B.; Voelcker-Rehage, C. Training Effects on Motor-Cognitive Dual-Task Performance in Older Adults. Eur Rev Aging Phys Act 2014, 11, 5-24, doi:10.1007/s11556-013-0122-z.

21. Uijen, I.L.; Aaronson, J.A.; Karssemeijer, E.G.A.; Olde Rikkert, M.G.M.; Kessels, R.P.C. Individual Differences in the Effects of Physical Activity on Cognitive Function in People with Mild to Moderate Dementia. JAD 2020, 74, 435-439, doi:10.3233/JAD190606.

22. Cordes, T.; Schoene, D.; Kemmler, W.; Wollesen, B. Chair-Based Exercise Interventions for Nursing Home Residents: A Systematic Review. J. Am. Med. Dir. Assoc 2020, doi:10.1016/j.jamda.2020.09.042.

23. Littbrand, H.; Stenvall, M.; Rosendahl, E. Applicability and Effects of Physical Exercise on Physical and Cognitive Functions and Activities of Daily Living among People with Dementia: A Systematic Review. Am J Phys Med Rehabil 2011, 90, 495-518, doi:10.1097/PHM.0b013e318214de26.

24. van Alphen, H.J.M.; Hortobágyi, T.; van Heuvelen, M.J.G. Barriers, Motivators, and Facilitators of Physical Activity in Dementia Patients: A Systematic Review. Arch Gerontol Geriatr 2016, 66, 109-118, doi:10.1016/j.archger.2016.05.008.

25. Eggenberger, E.; Heimerl, K.; Bennett, M.I. Communication Skills Training in Dementia Care: A Systematic Review of Effectiveness, Training Content, and Didactic Methods in Different Care Settings. Int Psychogeriatr 2013, 25, 345-358, doi:10.1017/S1041610212001664.

26. Travers, C.; Brooks, D.; Hines, S.; O’Reilly, M.; McMaster, M.; He, W.; MacAndrew, M.; Fielding, E.; Karlsson, L.; Beattie, E. Effectiveness of Meaningful Occupation Interventions for People Living with Dementia in Residential Aged Care: A Systematic Review. JBI Evidence Synthesis 2016, 14, 163-225, doi:10.11124/JBISRIR-2016-003230.

27. Eldridge, S.M.; Chan, C.L.; Campbell, M.J.; Bond, C.M.; Hopewell, S.; Thabane, L.; Lancaster, G.A. CONSORT 2010 Statement: Extension to Randomised Pilot and Feasibility Trials. BMJ 2016, i5239, doi:10.1136/bmj.i5239.

28. Cordes, T.; Bischoff, L.L.; Schoene, D.; Schott, N.; Voelcker-Rehage, C.; Meixner, C.; Appelles, L.-M.; Bebenek, M.; Berwinkel, A.; Hildebrand, C.; et al. A Multicomponent Exercise Intervention to Improve Physical Functioning, Cognition and Psychosocial Well-Being in Elderly Nursing Home Residents: A Study Protocol of a Randomized Controlled Trial in the PROCARE (Prevention and Occupational Health in Long-Term Care) Project. BMC Geriatr 2019, 19, doi:10.1186/s12877-0191386-6.

29. GKV-Spitzenverband Leitfaden Prävention in Stationären Pflegeeinrichtungen Nach § 5 SGB XI; BBGK Berliner Botschaft, Gesellschaft für Kommunikation $\mathrm{mbH}$, 2018;

30. Wollesen, B.; Argubi-Wollesen, A. Mobil \& Vital im Alter und bei Demenz Bewegungskompetenzen fördern und erhalten bei Senioren und Menschen mit demenziellen Erkrankungen. Ein Praxisbuch mit Übungsanregungen; Books on Demand: Norderstedt, 2012; ISBN 978-3-8448-2535-0.

31. Garber, C.E.; Blissmer, B.; Deschenes, M.R.; Franklin, B.A.; Lamonte, M.J.; Lee, I.-M.; Nieman, D.C.; Swain, D.P. Quantity and Quality of Exercise for Developing and Maintaining Cardiorespiratory, Musculoskeletal, and Neuromotor Fitness in Apparently Healthy Adults: Guidance for Prescribing Exercise. Med. Sci. Sports Exerc 2011, 43, 1334-1359, doi:10.1249/MSS.0b013e318213fefb.

32. Temple, R.O.; Stern, R.A.; Latham, J.; Ruffolo, J.S.; Arruda, J.E.; Tremont, G. Assessment of Mood State in Dementia by Use of the Visual Analog Mood Scales (VAMS). Am J Geriatr Psychiatry 2004, 12, 527-530, doi:10.1097/00019442-200409000-00012. 
33. Hendriks, I.; Meiland, F.J.M.; Gerritsen, D.L.; Dröes, R.-M. Evaluation of the 'Unforgettable' Art Programme by People with Dementia and Their Care-Givers. Ageing Soc 2021, 41, 294-312, doi:10.1017/S0144686X19001089.

34. Jørgensen, L.B.; Thorleifsson, B.M.; Selbæk, G.; Šaltytė Benth, J.; Helvik, A.-S. Physical Diagnoses in Nursing Home Residents - Is Dementia or Severity of Dementia of Importance? BMC Geriatr 2018, 18, doi:10.1186/s12877-018-0943-8. 